\title{
Occurrence of Ulcerative Colitis in a Patient With Adult-Onset Still's Disease: Coincidence or Correlated
}

\author{
Yun Jung CHOI, ${ }^{1}$ Wan-hee $\mathrm{YOO}^{2}$ \\ ${ }^{1}$ Department of Internal Medicine, Chonbuk National University Hospital, Division of Rheumatology, Jeonju, South Korea \\ ${ }^{2}$ Department of Internal Medicine, Chonbuk National University Medical School, Division of Rheumatology, Jeonju, South Korea
}

\begin{abstract}
A 37-year-old male patient with adult-onset Still's disease (AOSD) developed ulcerative colitis (UC) during the course of treatment. He complained of abdominal pain, diarrhea, and frequent passage of blood-stained stool with peri-umbilical tenderness. The laboratory evaluation was significant only for a hemoglobin level of $11 \mathrm{~g} / \mathrm{dL}$. The patient underwent endoscopic and colonoscopic examination with the presumptive diagnosis of nonsteroidal anti-inflammatory drug-induced gastropathy. Unexpectedly, the colonoscopic and pathologic findings of the biopsy specimen were compatible with UC. To our knowledge, this is the first report of AOSD concurrently occurring with UC. In patients with AOSD, abdominal pain is likely to be mistaken for nonsteroidal anti-inflammatory drug-induced gastropathy. However, several studies imply the possibility of UC as an extraarticular manifestation of AOSD. Although coexistence of AOSD and inflammatory bowel disease is rare, timely diagnosis is important since this co-occurrence may aggravate this otherwise benign disease.

Keywords: Abdominal pain; adult-onset Still's disease; inflammatory bowel disease; juvenile idiopathic arthritis; ulcerative colitis.
\end{abstract}

Adult-onset Still's disease (AOSD) is an autoinflammatory disorder, characterized by spiking fevers, evanescent salmon-pink rash, arthritis or arthralgia, and hyperleukocytosis. ${ }^{1}$ The pathogenesis remains unknown, but infection may be a trigger in a genetically susceptible host. Elevated levels of cytokines including interleukin-1, interleukin-6, and tumor necrosis factor alpha (TNF- $\alpha$ ) may play a key role in the induction and development of AOSD. ${ }^{2}$ Inflammatory bowel disease (IBD), comprising Crohn's disease (CD) and ulcerative colitis (UC), is a chronic inflammatory disease affecting the small intestine and colon. Multiple pathogenic factors including environmental, genetic, and gut microbiota interact with the immune system, leading to a dysregulated immune response, resulting in chronic intestinal inflammation. ${ }^{3}$ Inflammatory bowel disease is an extra-articular manifestation in rheumatic diseases such as spondyloarthropathies, but only a few cases on concurrent AOSD and IBD, mainly $\mathrm{CD}$, have been reported. In this report, we describe the first case of UC diagnosed during the treatment of AOSD.

\section{CASE REPORT}

A 37-year-old male patient was admitted for a seven-day history of fever above $39{ }^{\circ} \mathrm{C}$. He complained of headache, sore throat, and symmetric arthralgia of the shoulders and knees. Physical examination showed a salmon-colored macular rash on his abdomen and chest. Laboratory analysis revealed a white blood cell count of 27,000 cells $/ \mathrm{mm}^{3}$ (normal: 4,800-10,800 cells $/ \mathrm{mm}^{3}$ ), erythrocyte sedimentation rate of $108 \mathrm{~mm} /$ hour (normal $<9 \mathrm{~mm} /$ hour), C-reactive protein of $215 \mathrm{mg} / \mathrm{L}$ (normal $<5 \mathrm{mg} / \mathrm{L}$ ), aspartate aminotransferase 

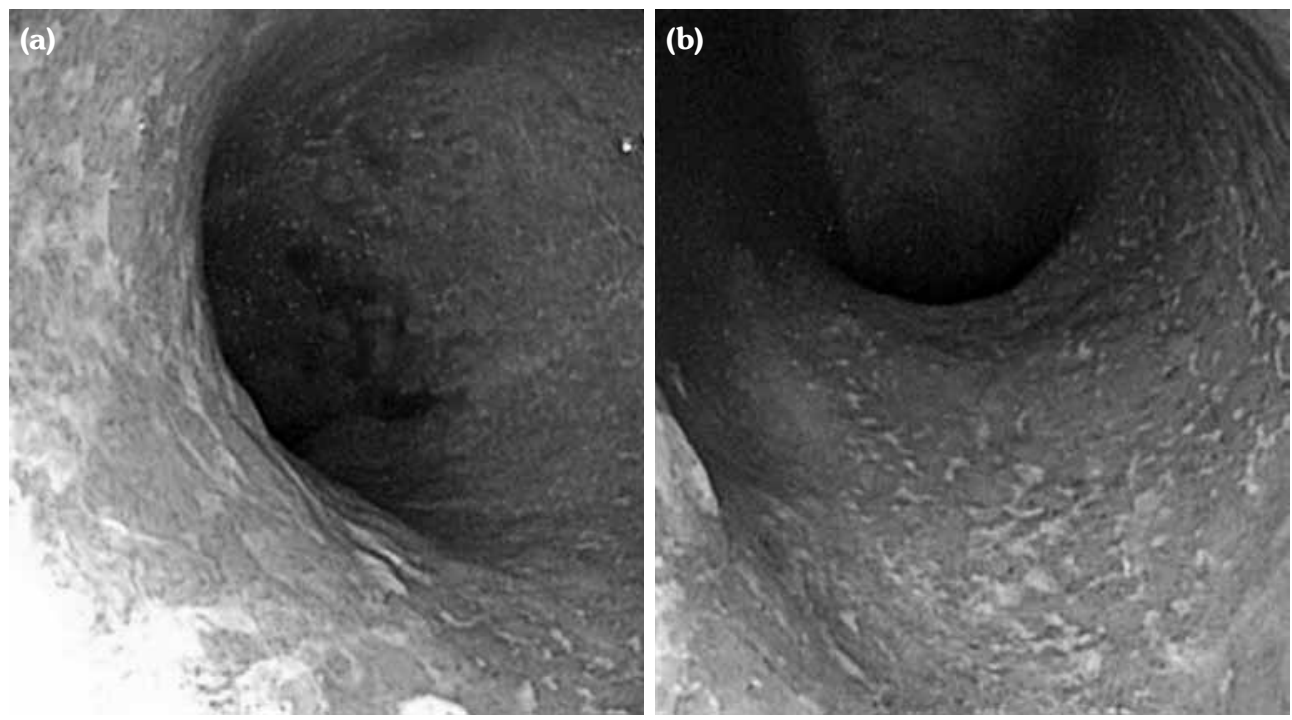

Figure 1. Colonoscopy shows diffuse erosion and multiple shallow ulcers (a) in sigmoid colon and (b) rectum.

level of $93 \mathrm{IU} / \mathrm{L}$ (normal $<33 \mathrm{IU} / \mathrm{L}$ ), and alanine aminotransferase level of $100 \mathrm{IU} / \mathrm{L}$ (normal $<35 \mathrm{IU} / \mathrm{L}$ ). The rest of the laboratory parameters were within normal limits. Antinuclear antibody, rheumatoid factor, and anti-cyclic citrullinated peptide antibody were all absent. Extensive evaluation to identify the cause of the fever revealed no evidence of infection or malignancy. However, his findings were indicative of AOSD. He complied with three major Yamaguchi criteria 4 fever, arthralgia, and leukocytosis-and several minor criteria including sore throat, negative rheumatoid factor and fluorescent antinuclear antibody test results, and abnormal liver function tests; these were also indicative of the diagnosis of AOSD. He was treated with nonsteroidal anti-inflammatory drugs (NSAIDs) and glucocorticoids $(0.5 \mathrm{mg} / \mathrm{kg} /$ day $)$, with symptom resolution and normalization of biochemical markers. After reduction of the steroids over a six-month period with concomitant administration of methotrexate $(12.5 \mathrm{mg} /$ week) and NSAIDs, the AOSD remained stable without relapse.
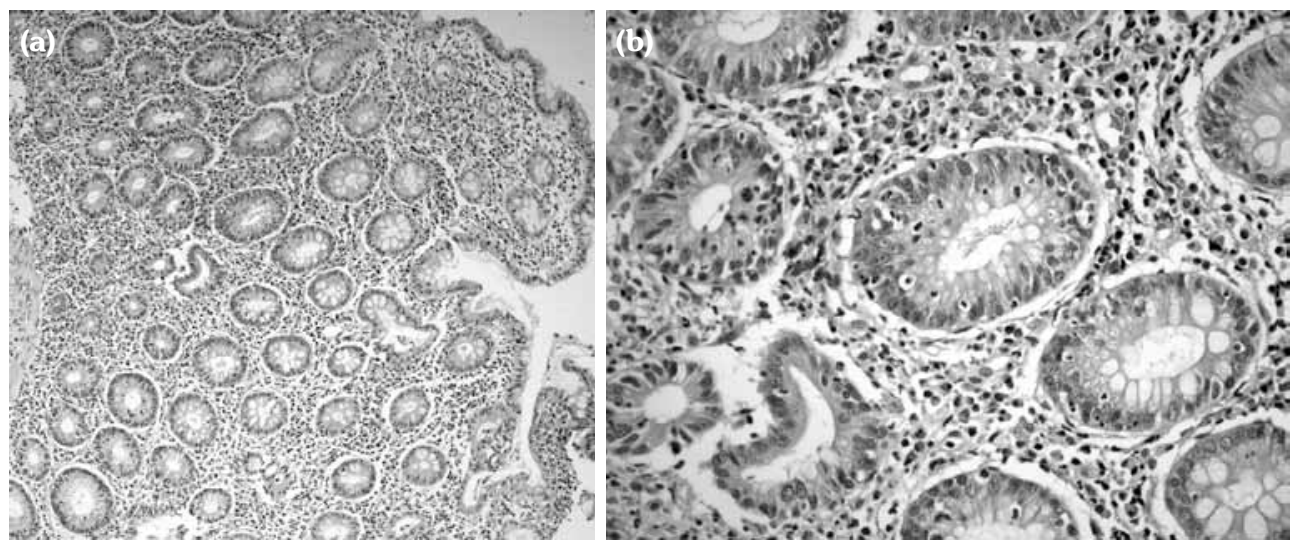

Figure 2. Light microscopy image of a colonoscopic biopsy with hematoxylin and eosin staining demonstrates glandular distortion (branching), increased lymphoplasma cells in lamia propria (a) $\mathrm{H}-\mathrm{E} \times 100$ ), and cryptitis, with neutrophils infiltrating lamina propria and crypt epithelium (b) $\mathrm{H}-\mathrm{E} \times 400$ ). 
After one year, he complained of abdominal pain, diarrhea, and frequent passage of bloodstained stool. Upon physical examination, mild tenderness was elicited in the peri-umbilical region. Laboratory evaluation was significant only for a hemoglobin level of $11 \mathrm{~g} / \mathrm{dL}$. The patient underwent endoscopic and colonoscopic examination with the presumptive diagnosis of NSAID-induced gastropathy. Unexpectedly, diffuse erosion with edema and erythema, and multiple shallow ulcers were detected in the sigmoid colon and rectum by colonoscopy (Figure 1). The mucosal biopsy specimens revealed glandular distortion and cryptitis with crypt abscess (Figure 2), which were compatible with UC. After establishing the diagnosis of UC, he was treated with hydrocortisone and mesalazine. His symptoms of colitis began to improve after two weeks of treatment.

\section{DISCUSSION}

Adult-onset Still's disease presents with heterogeneous manifestations. However, no cases on the coexistence of AOSD and UC have been reported to date, whereas $\mathrm{CD}$ with AOSD is occasionally reported. The mechanisms underlying the intestinal-related presentation of AOSD remain obscure, but various data supporting the association between arthritis and IBD have been reported. For example, a study including 792 patients with IBD revealed that a considerable portion of those with $\mathrm{CD}$ (20.2\%) and UC (11.0\%) present with arthritis. ${ }^{5}$ In a study in Finland, pediatric-onset IBD patients showed a higher frequency of arthritis compared with the control group $(5.4 \%$ versus $0.2 \%){ }^{6}$ Furthermore, in an analysis of patients with juvenile idiopathic arthritis (JIA), $81 \%$ of the patients showed abnormal findings that were classified as active chronic or CD-like lesions on ileocolonoscopy. ${ }^{7}$ Additionally, the polymorphisms for JIA have been shown to share with those of other autoimmune diseases including $\mathrm{CD}$ in the genome-wide association studies. ${ }^{8}$ AOSD follows the same continuum as systemic-onset JIA, so it is regarded as a subtype of JIA. In addition, recent studies suggest that AOSD has two distinct subsets: one with prominent systemic features and the other with chronic arthritis associated with TNF- $\alpha .{ }^{9}$ In light of this, AOSD, as a subtype of JIA or arthritis, could exhibit IBD as an extra-articular manifestation. This may be the supporting evidence for the unusual cases of coexistence of AOSD and IBD. Anti-TNF- $\alpha$ antibodies have been used in the treatment of both AOSD and refractory IBD, because TNF- $\alpha$ is implicated in the pathogenesis of both of them. Considering this information, it is plausible that IBD could be present concurrently with AOSD as an extra-articular manifestation.

It is known that inflammatory-related extraintestinal manifestations are more common in $\mathrm{CD}$ than in UC and a more vigorous response and increased $\mathrm{T}$ cell function occur in $\mathrm{CD}$ than in UC. However, since both $\mathrm{CD}$ and UC share multiple pathologic factors including environmental changes, abnormal gut microbiota, and a wide aberrant immune response ${ }^{3}$ it is possible to see the coexistence of AOSD and UC, as in our case.

Diagnosis of AOSD is challenging for the clinicians. It presents diverse manifestations that are not disease specific; clinicians need to exclude neoplastic, infectious, or inflammatory disorders first. After establishing the diagnosis, heterogeneous manifestations are not easily distinguishable, whether originating from the AOSD or other complications. For example, when patients with AOSD complain of abdominal discomfort, they are likely to have a presumptive diagnosis of NSAID-induced gastropathy because the treatment of AOSD has centered on the use of NSAIDs. However, as seen in our case, UC may be a causative factor for abdominal pain, so the routine empirical treatment without detailed investigation may aggravate the disease. Accordingly, we believe that patients' personal history and laboratory and endoscopic evaluation results may provide the exact and timely diagnosis and management of UC combined with AOSD.

\section{Declaration of conflicting interests}

The authors declared no conflicts of interest with respect to the authorship and/or publication of this article.

\section{Funding}

The authors received no financial support for the research and/or authorship of this article. 


\section{REFERENCES}

1. Efthimiou P, Paik PK, Bielory L. Diagnosis and management of adult onset Still's disease. Ann Rheum Dis 2006;65:564-72.

2. Efthimiou P, Georgy S. Pathogenesis and management of adult-onset Still's disease. Semin Arthritis Rheum 2006;36:144-52.

3. de Souza HS, Fiocchi C. Immunopathogenesis of IBD: current state of the art. Nat Rev Gastroenterol Hepatol 2016;13:13-27.

4. Yamaguchi M, Ohta A, Tsunematsu T, Kasukawa R, Mizushima Y, Kashiwagi H, et al. Preliminary criteria for classification of adult Still's disease. J Rheumatol 1992;19:424-30.

5. Veloso FT, Carvalho J, Magro F. Immune-related systemic manifestations of inflammatory bowel disease. A prospective study of 792 patients. J Clin
Gastroenterol 1996;23:29-34.

6. Turunen P, Ashorn M, Auvinen A, Iltanen S, Huhtala $\mathrm{H}$, Kolho KL. Long-term health outcomes in pediatric inflammatory bowel disease: a population-based study. Inflamm Bowel Dis 2009;15:56-62.

7. Mielants H, Veys EM, Joos R, Cuvelier C, De Vos M, Proot F. Late onset pauciarticular juvenile chronic arthritis: relation to gut inflammation. J Rheumatol 1987;14:459-65.

8. Thompson SD, Sudman M, Ramos PS, Marion MC, Ryan M, Tsoras M, et al. The susceptibility loci juvenile idiopathic arthritis shares with other autoimmune diseases extend to PTPN2, COG6, and ANGPT1. Arthritis Rheum 2010;62:3265-76.

9. Jamilloux Y, Gerfaud-Valentin M, Martinon F, Belot A, Henry T, Sève P. Pathogenesis of adult-onset Still's disease: new insights from the juvenile counterpart. Immunol Res 2015;61:53-62. 